\title{
Retardo estatural em menores de cinco anos: um estudo "baseline"
}

\author{
Linear growth retardation in children under five years of age: \\ a baseline study
}

\author{
Anete Rissin ${ }^{1}$ \\ José $N$ atal Figueiroa ${ }^{1}$ \\ M aria Helena D'Aquino Benício ${ }^{2}$ \\ $M$ alaquias Batista Filho ${ }^{1}$
}

${ }^{1}$ Departamento de Pesquisas, Instituto de $M$ edicina Integral Prof. Fernando Figueira - IM IP. Rua dos Coelhos, $n=300$. 50070-550 Recife PE. arissin@gmail.com

${ }^{2}$ FaculdadedeSaúde Pública, Universidade de São Paulo
Abstract The scope of this study was to describe the prevalence of, and analyze factors associated with, linear growth retardation in children. The baseline study analyzed 2040 children under the age of five, establishing a possible association be tween growth delay (height/age index $\leq 2$ scores $Z)$ and variables in six hierarchical blocks: socioeconomic, residence, sanitary, maternal, biological and healthcare access. M ultivariate analysis was performed using Poisson regression with the robust standard error option, obtaining adjusted prevalenceratios with a $\mathrm{Cl}$ of $95 \%$ and the respective significant probability values. Among nonbinary variables, there was a positive association with roof typeand number of inhabitants per room and a negative association with income per capita, mother's schooling and birth weight. The adjusted analysis also indicated water supply, visit from the community health agent, birth delivery location, internment for diarrhea, or for pneumonia and birth weight as significant variables. Several risk factors were identified for linear growth retardation pointing to the multi-causal aspects of the problem and highlighting the need for control measures by the various hierarchical government agents.

Key words Linear growth retardation, M alnutrition, Multivariate analysis, Hierarchical model
Resumo Objetivo: Descrever a prevalência eanalisar fatores associados ao retardo estatural em menores de cinco anos. M étodos: Estudo "baseline", que analisou 2.040 crianças, verificando possíveis associações entre o retardo estatural (índice altura/idade $\leq 2$ escores Z) e variáveis hierarquizadas em seis blocos: socioeconômicas, do domićli io, do saneamento, maternas, biológicas e de acesso aos serviços de saúde. A análise multivariada foi realizada por regressão de Poisson, com opção de erro padrão robusto, obtendo-se as razões de prevalência ajustadas, com IC 95\% erespectivos val ores de significância. Resultados: Entre as variáveis não dicotômicas, houve associação positiva com o tipo de teto e o número de moradores por cômodo e associação negativa com renda, escolaridade da mãe e peso ao nascer. A análiseajustada indicou ainda como variáveissignificantes: abastecimento deágua, visita do agente comunitário de saúde, local do parto, internação por diarreia e internação por pneumonia. Conclusão: Os fatores identificados como de risco para o retardo estatural configuram a multicausalidade do problema, implicando na necessidade de intervenções multisetoriais e multiníveis para o seu controle.

Palavras-chave Retardo estatural, D esnutrição, Análise multivariada, M odelo hierarquizado 
Introdução

A desnutrição energético-proteica (DEP) ainda figura como um dos principais problemas de saúde das crianças no mundo, afetando estimativamente $29 \%$ dos menores de cinco anos, com prevalências que variam de $8 \%$ a $10 \%$ na América Latina $^{1}$. Destacadamente, na Ásia M eridional e África Subsaariana, a DEP representada pelo retardo estatural, afetaria, respectivamente $29,9 \%$ e $33,8 \%$ dos menores de cinco anos, associando-se a $60 \%$ das mortes nesta faixa etária e produzindo uma série de outros efeitos biológicos e sociais, entre os quais déficits no desempenho mental ${ }^{2}$, predisposição a importantes doenças crônicas não transmissíveis e outros riscos adicionais à saúde, os quais podem se prolongar por toda vida.

No Brasil, o problema da DEP apresentou marcantes reduções nas últimas três décadas, decaindo de uma prevalência inicial de 37,0\% (1974/ $1975)^{3}$ para $19,8 \%(1989)^{4}, 13,5 \%(1996)^{5}$ e, finalmente, $6,8 \%(2006)^{6}$. Ao lado desses marcos temporais para o conjunto do país, foram evidenciadas substanciais reduções de ocorrências nas regiões N orte e N ordeste, as mais pobres do país. 0 estado dePernambuco, no Nordestebrasileiro, dispõe de uma situação ímpar entre as demais unidades federativas do Brasil, na medida em que conta com três inquéritos de base populacional estatisticamente representativos da situação de saúde materno-infantil edo estado nutricional das crianças: $1991^{7}, 1997^{8}$ e2006. Estes doisúltimos (II ellI Pesquisa Estadual de Saúde e Nutrição) configuram um extenso e variado banco de dados, com detaIhamento aprofundado do estado nutricional (antropometria, dados clínicos e laboratoriais) de crianças emulheres no período reprodutivo, no contexto de variáveis sobre condições ambientais, ren$\mathrm{da}$, escolaridade, doenças prevalentes, acesso a serviços básicos (educação, saúde, rede de proteção social), representando, assim, possibilidades de uma análise mais completa e integrada dos múltiplos fatores que intervêm na gênese da DEP.

Por outro lado, tendo em vista a rapidez com que se desenvolve o processo de transição nutricional no Brasil e, principalmente no Nordeste, em anos mais recentes, destacadamente na década de $90^{10,11}$, torna-se oportuno, relevante e pertinenteestabelecer uma base comparativa da pre valência e, sobretudo, dos fatores de risco como referência temporal para acompanhar e entender o desenvolvimento prospectivo do processo em sua etapa mais dinâmica. São estes, portanto, os objetivos que justificam o presente estudo e seus próximos desdobramentos.
Método

Foi avaliada uma amostra de 2.040 crianças me nores de cinco anos, envolvidas na II Pesquisa Estadual sobre Saúde eN utrição (II PESN ), realizada no ano de 1997, abrangendo 18 municípios do estado de Pernambuco, dos quais cinco na Região $M$ etropolitana do Recifee 13 no interior do estado. Constituiu-senum inquérito debase domiciliar, tipo transversal, tendo como propósito descrever a condição denutrição ede saúde da população pernambucana, com ênfase no grupo materno-infantil. Neste estudo, a variável resposta foi o retardo estatural das crianças menores de cinco anos, expresso pela relação altura/idade (A/ I), e classificado segundo a dicotomia desnutrido/eutrófico. Considerou-se como retardo estatural a criança com - 2 escores - $z$ do índice A/I, tendo como padrão dereferência a tabela $\mathrm{NCH} \mathrm{S}^{12}$. Este padrão foi adotado levando-se em conta que os dados aqui analisados devem ser comparados com outros relatos publicados na década de 90 em Pernambuco $\left(1991^{7}, 1996^{8}\right)$ e em outros estados do Nordeste que também o utilizaram.

As variáveis explanatórias foram agrupadas em seis grupos hierarquicamente ordenados: 1) variáveis socioeconômicas; 2) características físicas eocupacionais da habitação; 3) variáveis referentes ao saneamento; 4) variáveis maternas; 5) variáveis ligadas ao acesso aos serviços de saúde; 6) variáveis diretamente relacionadas à criança, representando o nível mais proximal do modelo. 0 ideograma analítico, apresentado na Figura 1, adotou 0 enfoque hierárquico, partindo de um modelo conceitual de referência, a partir dehipóteses sobre fatores causais e sua sequência lógica na história "natural" do problema. Assume-se como pressuposto que os fatores condicionantes podem agir direta ou indiretamente sobre o estado nutricional das crianças, implicando em diferentes níveis hierárquicos de determinação 2,13.

Como etapa prévia à análise multivariada, foram feitas análises bivariadas, selecionando-se as que apresentavam um valor $p<0,20$. As análises multivariadas foram processadas através do ajuste de modelos de regressão de Poisson, com erro padrão robusto, estimando-se as razões de prevalência (RP), com respectivos intervalos de confiança de $95 \%$ e os níveis de significância. A significância estatística de cada variável foi calculada através do teste de Wald para heterogeneidade ou tendência linear, conforme o caso. Os níveis hierárquicos foram compostos pelas variáveis que apresentaram um valor $p<0,20$ na análise bivariada. As variáveis sexo e idade fo- 


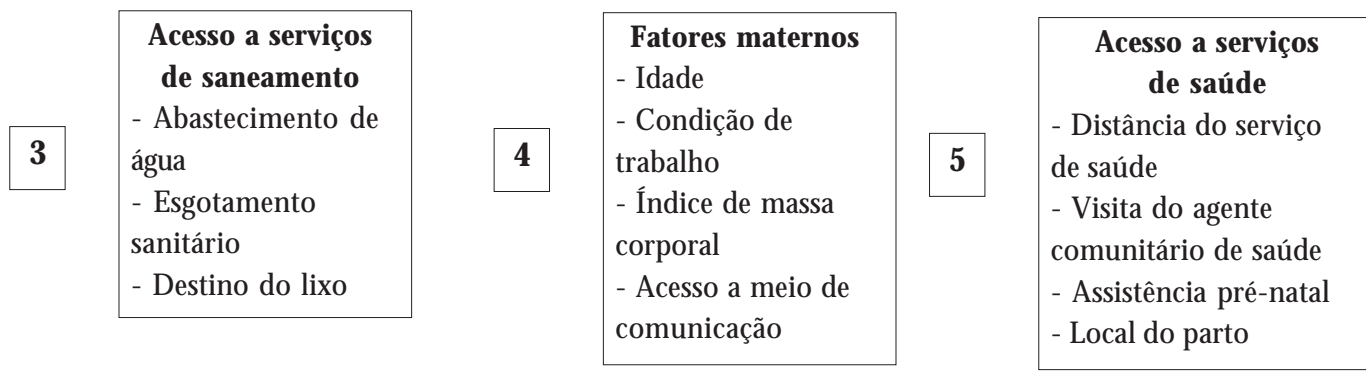

\begin{tabular}{|l|l|}
\multicolumn{1}{|c|}{ Fatores ligados à criança } \\
\hline 6 & $\begin{array}{l}\text { - Peso ao nascer } \\
\text { - Internação por diarreia (nos últimos } 12 \text { meses) } \\
\text { - Internação por pneumonia (nos últimos } 12 \text { meses) }\end{array}$ \\
& $\begin{array}{l}\text { Fatores biológicos } \\
\text { - Sexo } \\
\text { - Idade }\end{array}$ \\
\hline Retardo estatural em menores de cinco anos \\
\hline
\end{tabular}

Figura 1. M odelo conceitual hierarquizado de determinação do retardo estatural em menos de cinco anos, no estado de Pernambuco.

ram introduzidas no modelo como controle, em função de seu significado biológico e pela condição de poderem interferir diretamente na determinação do problema, não recebendo influência de outras variáveis.

A II PESN foi apoiada pelo M inistério da Saúde (Secretaria de Ciência eTecnologia) epela Secretaria de Saúde do Estado dePernambuco, tendo sido oportunamente informada e consensuada junto aos seus coordenadores regionais de saúde para sua legitimação institucional, suporte logístico, apoio político e requisitos éticos. As famílias entrevistadas foram informadas acerca dos objetivos da pesquisa e sobre o caráter sigiloso dos dados. Assim, as medidas antropomé tricas, os dados anamnéticos e os exames complementares de laboratório das crianças meno- res de cinco anos só foram efetuados mediante autorização prévia dos pais e/ou responsáveis. O utras informações mais genéricas sobre o estudo, o trabalho de campo, a organização e as provas de consistência do banco de dados acham-se relatadas em publicação anterior ${ }^{5}$.

0 estudo utilizou o banco de dados da II Pesquisa Estadual deSaúde eN utrição, apoiada pelo M inistério da Saúdee pela Secretaria Estadual de Saúde de Pernambuco.

\section{Resultados}

Os resultados exibidos na Tabela 1 mostram que, com exceção do sexo, todas as variáveis estudadas apresentaram associação com o retardo es- 
tatural, no nível de significância adotado para este estágio da análise estatística. O bserva-se que a prevalência de retardo estatural se elevou, sucessivamente, de 7,4\% (renda $\geq 0,5$ e $<1,0$ s.m.), para $16,2 \%$ (renda $\geq 0,25 \mathrm{e}<0,5$ ), alcançando $18,3 \%$ quando a renda familiar per capita se si- tuava abaixo de 0,25 s.m. Nas crianças filhas de mães sem instrução, a prevalência de retardo estatural foi de $18,8 \%$, contrastando significativamente com a situação de crianças de mães com um ou mais anos de escolaridade. Os fatores re lacionados com os diversos itens que descrevem

Tabela 1. Retardo estatural de menores de cinco anos, segundo fatores biológicos e socioeconômicos. Pernambuco, 1997.

\begin{tabular}{|c|c|c|c|c|c|}
\hline \multirow[t]{2}{*}{ Variável } & \multirow{2}{*}{$\begin{array}{c}\text { Amostra } \\
n\end{array}$} & \multicolumn{2}{|c|}{$\begin{array}{l}\text { Retardo } \\
\text { estatural }\end{array}$} & \multirow{2}{*}{ RP (IC95\%) } & \multirow{2}{*}{ Valor $p$} \\
\hline & & $n$ & $\%$ & & \\
\hline Sexo & 2040 & & & & 0,835 \\
\hline Feminino & 1028 & 126 & 12,3 & 1,0 & \\
\hline Masculino & 1012 & 121 & 12,0 & 0.98 (0.77 a 1.23$)$ & \\
\hline Idade (meses) & 2040 & & & & \\
\hline 0 a 11 & 450 & 49 & 10,9 & 1,0 & 0,043 \\
\hline 12 a 23 & 408 & 64 & 15,7 & 1.44 (1.02 a 2.04$)$ & \\
\hline 24 a 59 & 1182 & 134 & 11,3 & $1.04(0.76$ a 1.42$)$ & \\
\hline Renda familiar per capita & 2022 & & & & $<0,001^{\top}$ \\
\hline$>1,00$ & 424 & 10 & 2,4 & 1,0 & \\
\hline$>0,50$ e $<1,00$ & 408 & 30 & 7,4 & $3,12(1,54$ a 6,30$)$ & \\
\hline$>0,25$ e $<0,50$ & 594 & 96 & 16,2 & 6.85 (3.62 a 12.99) & \\
\hline$<0,25$ & 596 & 109 & 18,3 & $7,75(4,11$ a 14,64$)$ & \\
\hline Escolaridade da mãe (anos) & 2025 & & & & $<0,001$ \\
\hline$>4$ & 840 & 158 & 18,8 & 1,0 & \\
\hline 1 a 3 & 645 & 55 & 8,5 & $2,12(1,59$ a 2,82$)$ & \\
\hline Sem instrução & 540 & 32 & 5,9 & $3,19(2,42$ a 4,22$)$ & \\
\hline Tipo de parede & 2040 & & & & 0,001 \\
\hline Alvenaria ou tijolo & 1748 & 193 & 11,0 & 1,0 & \\
\hline Outros & 292 & 54 & 18,5 & 1.67 (1.27 a 2.20$)$ & \\
\hline Tipo de piso & 2040 & & & & \\
\hline Cerâmica & 344 & 12 & 3,5 & 1,0 & $<0,001^{\top}$ \\
\hline Cimento & 1411 & 193 & 13,7 & $3.92(2.21$ a 6.94$)$ & \\
\hline Tijolo & 285 & 42 & 14,7 & $4.22(2.27$ a 7.87$)$ & \\
\hline Tipo de teto & 2040 & & & & \\
\hline Laje & 317 & 10 & 3,2 & 1,0 & $<0,001^{\top}$ \\
\hline Telha & 1530 & 206 & 13,5 & 4.27 (2.29 a 7.96$)$ & \\
\hline Outros & 193 & 31 & 16,1 & 5.09 (2.55 a 10.15$)$ & \\
\hline $\mathrm{N} \cong$ de moradores por cômodo & 2040 & & & & \\
\hline$<1$ & 415 & 18 & 4,3 & 1,0 & $<0,001^{\top}$ \\
\hline 1 a menos de 2 & 1003 & 118 & 11,8 & $2.72(1.68$ a 4.40$)$ & \\
\hline 2 a menos de 3 & 340 & 52 & 15,3 & 3.60 (2.18 a 5.94$)$ & \\
\hline$>3$ & 282 & 59 & 20,9 & 5.33 (3.16 a 8.99) & \\
\hline Abastecimento de água & 2040 & & & & \\
\hline Rede geral & 1142 & 90 & 7,9 & 1,0 & $<0,001$ \\
\hline Outros & 898 & 157 & 17,5 & 2.22 (1.74 a 2.83$)$ & \\
\hline Esgotamento sanitário & 2040 & & & & \\
\hline Rede pública & 539 & 31 & 5,8 & 1,0 & $<0,001$ \\
\hline Fossa com tampa & 723 & 79 & 10,9 & 1.90 (1.27 a 2.83$)$ & \\
\hline Ausente & 778 & 137 & 17,6 & 3.06 (2.11 a 4.45$)$ & \\
\hline Tratamento da água de beber & 2040 & & & & \\
\hline Fervida ou mineral ou filtrada ou clorada & 1186 & 97 & 8,2 & 1,0 & $<0,001$ \\
\hline Coada ou sem tratamento ou outro & 854 & 150 & 17,6 & $2.15(1.68 \mathrm{a} \quad 2.73)$ & \\
\hline Destino do lixo & 2040 & & & & \\
\hline Coleta pública & 1030 & 84 & 8,2 & 1,0 & $<0,001$ \\
\hline Outra forma & 1010 & 163 & 16,1 & 1.98 (1.54 a 2.54$)$ & \\
\hline
\end{tabular}

$\mathrm{T}=$ " $\mathrm{p}$ " detendência linear. $\mathrm{RP}=$ razão de prevalência. 
as condições físicas das habitações, serviços básicos de saneamento e cuidados higiênicos com a água de beber, se apresentaram estatisticamente significantes em relação à probabilidade de ocorrência de déficit estatural.

Asvariáveis maternas (idade, condição detrabalho e índice de massa corporal) não se apresentaram estatisticamente associadas à ocorrência do déficit estatural das crianças. Já a variável que representa 0 acesso à meio de comunicação (TV), as referentes a serviços de saúde e as relativas à criança, achavam-se estatisticamente associadas ao retardo estatural (Tabela 2).
$\mathrm{Na}$ Tabela 3 estão detalhados os resultados dos modelos ajustadosa partir de análises bivariadas selecionadas nas Tabelas 1 e 2. Verifica-se que a renda familiar ea escolaridade das mães permaneceram no modelo final, com pequenas reduções em relação aos valores das razões brutas. Também se mantiveram estatisticamente significantes as correlações com o tipo de teto, o número de moradores por cômodo, o abastecimento de água, o local do parto, a visita do agente comunitário de saúde, o peso ao nascer, a internação por diarreia e a internação por pneumonia.

Tabela 2. Retardo estatural de menores de cinco anos, pela relação altura/idade, segundo fatores: maternos e referentes a serviços de saúde e à criança. Pernambuco, 1997.

\begin{tabular}{|c|c|c|c|c|c|}
\hline \multirow[t]{2}{*}{ Variável } & \multirow{2}{*}{$\begin{array}{c}\text { Amostra } \\
n\end{array}$} & \multicolumn{2}{|c|}{$\begin{array}{l}\text { Retardo } \\
\text { estatural }\end{array}$} & \multirow{2}{*}{ RP (IC95\%) } & \multirow{2}{*}{ Valor $\mathrm{p}$} \\
\hline & & $\mathrm{n}$ & $\%$ & & \\
\hline Idade da mãe & 2031 & & & & $0.173^{\top}$ \\
\hline 10 a menos de 20 & 124 & 11 & 8,9 & 1,0 & \\
\hline 20 a menos de 25 & 540 & 58 & 10,7 & $1.21(0.65$ a 2.24$)$ & \\
\hline 25 a menos de 35 & 950 & 126 & 13,3 & $1.50(0.83$ a 2.69$)$ & \\
\hline 35 ou mais & 417 & 51 & 12,2 & $1.38(0.74$ a 2.56$)$ & \\
\hline Condição de trabalho & 2027 & & & & 0.172 \\
\hline Trabalha & 776 & 84 & 10,8 & 1,0 & \\
\hline Não trabalha & 1251 & 161 & 12,9 & $0.84(0.65$ a 1.08$)$ & \\
\hline Índice de massa corporal & 1989 & & & & 0.872 \\
\hline$\geq 18.5$ & 1862 & 229 & 12,3 & 1,0 & \\
\hline$<18.5$ & 127 & 15 & 11,8 & $1.04(0.64$ a 1.70$)$ & \\
\hline Acesso a meio de comunicação (TV) & 2040 & & & & $<0,001$ \\
\hline $\mathrm{Sim}$ & 1637 & 166 & 10,1 & 1,0 & \\
\hline Não & 403 & 81 & 20,1 & 1.98 (1.56 a 2.53$)$ & \\
\hline Distância do serviço de saúde & 1952 & & & & $<0,001$ \\
\hline$\leq 2 \mathrm{~km}$ & 1458 & 143 & 9,8 & 1,0 & \\
\hline$>2 \mathrm{~km}$ & 494 & 88 & 17,8 & $1.82(1.42$ a 2.32$)$ & \\
\hline ACS (PACS ou Pastoral) & 2026 & & & & \\
\hline Recebe & 673 & 107 & 15,9 & 1,0 & 0,001 \\
\hline $\mathrm{N}$ ão recebe & 1353 & 140 & 10,3 & 0.65 (0.51 a 0.82$)$ & \\
\hline Assistência prénatal & 2000 & & & & \\
\hline Sim & 1603 & 163 & 10,2 & 1,0 & $<0,001$ \\
\hline Não & 397 & 82 & 20,7 & 2.03 (1.60 a 2.59$)$ & \\
\hline Local do parto & 2035 & & & & $<0,001$ \\
\hline Hospitalar & 1867 & 202 & 10,8 & 1,0 & \\
\hline Domiciliar ou outro & 168 & 45 & 26,8 & 2.48 (1.87 a 3.28$)$ & \\
\hline Peso ao nascer & 1875 & & & & $<0,001^{\top}$ \\
\hline 3000 ou mais & 1358 & 95 & 7,0 & 1,0 & \\
\hline 2500 a menos de 3000 & 368 & 65 & 17,7 & $2.52(1,88$ a 3,39$)$ & \\
\hline$<2500$ & 149 & 40 & 26,8 & $3,83(2,76$ a 5,33$)$ & \\
\hline Internação por diarréia & 2039 & & & & 0,007 \\
\hline Não & 1983 & 234 & 11,8 & 1,0 & \\
\hline Sim & 56 & 13 & 23,2 & $1,96(1,20$ a 3,22$)$ & \\
\hline Internação por pneumonia & 2039 & & & & 0,017 \\
\hline Não & 1973 & 233 & 11,8 & 1,0 & \\
\hline $\mathrm{Sim}$ & 66 & 14 & 21,2 & $1,71(1,11$ a 2,90$)$ & \\
\hline
\end{tabular}

$T=$ " $p$ " detendência linear. $\mathrm{RP}=$ razão de prevalência 
Tabela 3. Análise hierarquizada (regressão de Poisson) dos fatores associados ao retardo estatural em menores de cinco anos. Pernambuco, 1997.

\begin{tabular}{|c|c|c|c|c|c|}
\hline \multirow{2}{*}{$\begin{array}{c}\text { Variáveis } \\
\text { Renda familiar per capita (1) }\end{array}$} & \multirow{2}{*}{$\begin{array}{c}\% \text { de } \\
\text { retardo }\end{array}$} & \multirow[t]{2}{*}{ RP bruto (IC) } & \multicolumn{3}{|c|}{ RP ajustada } \\
\hline & & & $<0,001^{\top}$ & & $<0,001^{\top}$ \\
\hline$>1,00$ & 2,4 & 1,0 & & 1,0 & \\
\hline$<0,25$ & 18,3 & 7.75 (4.11 a 14.64$)$ & & $5.42(2.80$ a 0.53$)$ & \\
\hline$>0,25$ e $<0,50$ & 16,2 & 6.85 (3.62 a 12.99$)$ & & $5.21(2.70$ a 0.06$)$ & \\
\hline$\geq 0,50$ e $<1,00$ & 7,4 & $3.12(1.54$ a 6.30$)$ & & $2.70(1,33$ a 5,49$)$ & \\
\hline Escolaridade da mãe (anos) (1) & & & $<0,001$ & & $<0,001^{\top}$ \\
\hline Quatro ou mais & 7,4 & 1,0 & & 1,0 & \\
\hline De 1 a menos de 4 & 15,6 & $2.12(1.59$ a 2.82$)$ & & $1.46(1.09$ a 1.97$)$ & \\
\hline Sem instrução & 23,5 & $3,19(2,42$ a 4,22$)$ & & $2,06(1,53$ a 2,76$)$ & \\
\hline Tipo de teto (2) & & & $<0,001^{\top}$ & & $0.029^{\top}$ \\
\hline Laje & 3,2 & 1,0 & & 1,0 & \\
\hline Telha & 13,5 & $4,78(2,51$ a 9,09$)$ & & $1.72(0.91$ a 3.28$)$ & \\
\hline Outros & 16,1 & $5,87(2,81$ a 12,30$)$ & & $2.11(1.04$ a 4.28$)$ & \\
\hline № médio de moradores por cômodo (2) & & & $<0,001^{\top}$ & & $0.002^{\top}$ \\
\hline Menos de 1 & 4,3 & 1,0 & & 1,0 & \\
\hline De 1 a menos de 2 & 11,8 & $2,95(1,76$ a 4,93$)$ & & $1.71(1.04$ a 2.80$)$ & \\
\hline De 2 a menos de 3 & 15,3 & $4.07(2.37$ a 7.00$)$ & & 1.83 (1.07 a 3.12) & \\
\hline Três ou mais & 23,1 & 6.63 (3.64 a 12.09$)$ & & $2.45(1.40$ a 4.30$)$ & \\
\hline A bastecimento de água (3) & & & $<0,001$ & & 0.036 \\
\hline Rede geral & 7,9 & 1,0 & & 1,0 & \\
\hline Outros & 17,5 & 2.22 (1.74 a 2.83$)$ & & $1.33(1.02$ a 1.75$)$ & \\
\hline ACS (PACS ou Pastoral) (5) & & & & & 0.021 \\
\hline Recebe visita & 15,9 & 1,0 & 0,001 & 1,0 & \\
\hline Não recebe visita & 10,3 & $0,61(0,42$ a 0,82$)$ & & $0.76(0.60$ a 0.96$)$ & \\
\hline Local do parto (5) & & & $<0,001$ & & 0.001 \\
\hline Hospitalar & 10,8 & 1,0 & & 1,0 & \\
\hline Domiciliar ou outro & 26,8 & $3,02(2,08$ a 4,38$)$ & & $1.64(1.23$ a 2.20$)$ & \\
\hline Peso ao nascer (6) & & & $<0,001^{\top}$ & & $<0,001^{\top}$ \\
\hline 3000 ou mais & 7,0 & 1,0 & & 1,0 & \\
\hline 2500 a menos de 3000 & 17,7 & $2,85(2,01$ a 4,04$)$ & & $3.48(2.52$ a 4,82$)$ & \\
\hline$<2500$ & 26,8 & $4,88(3,17$ a 7,50$)$ & & $2,54(1,91$ a 3,39$)$ & \\
\hline Internação por diarréia (6) & & & 0.007 & & 0.043 \\
\hline Não & 11,8 & 1,0 & & 1,0 & \\
\hline Sim & 23,2 & $1.96(1.20$ a 3.22$)$ & & 1.70 (1.01 a 2.85$)$ & \\
\hline Internação por pneumonia (6) & & & 0.017 & & 0.048 \\
\hline Não & 11,8 & 1,0 & & 1,0 & \\
\hline Sim & 21,2 & $1.79(1,11$ a 2.90$)$ & & $1.71(1.00$ a 2.90$)$ & \\
\hline
\end{tabular}

$\mathrm{T}=$ " $\mathrm{p}$ " detendência linear; $\mathrm{T}=$ " $\mathrm{p}$ " linear trend; M odelo $1=$ renda familiar per capita + escolaridade materna; M odelo $2=$ M odelo1 + tipo de teto +número médio de moradores por cômodo; M odelo $3=$ M odelo 2; M odelo $4=$ M odelo 3; M odelo $5=$ M odelo 3 + distância ao serviço de saúde + visita do agente de saúde + local do parto; $M$ odel $06=$ M odelo $5+$ peso ao nascer; $M$ odel $1=$ per capita income + mother; $M$ odel $2=M$ odelo $1+$ roof + type of average number of residents per room; $M$ odel $3=$ M odel 2 ; $M$ odel $4=M$ odel 3 ; $M$ odel $5=M$ odel $3+$ distance to the health service agent's visit + health + place of birth; M odel $6=$ M odel $5+$ birthweight

\section{Discussão}

Deve ser considerado, em caráter preliminar, que este estudo deriva de um banco de dados não especificamente concebido para responder aos objetivos explícitos deste trabalho. Em outras palavras: não se idealizou, previamente, a construção deum model o hipotético causal da desnutrição expressa como "proxi" do retardo estatural de crianças menores de cinco anos. No entanto, no estudo de campo da II PESN, foram con- templadas informações que, para diferentes fins, podem ser ordenadas segundo a lógica estrutural defatoreshierarquizados $\varsigma^{2,13}$. Esta ordenação pressupõe, ao lado da lógica formal do modelo, um conhecimento prévio de cada problema a ser estudado em cada contexto, o que pode resultar em dificuldades, visto que, muitas vezes, não se dispõe de estudos e experiências anteriores como suporte empírico para justificar a escolha do modelo. Geralmente, os inquéritos nutricionais com propósitos analíticos obedecem a uma ma- 
triz genérica de hipóteses que atenderiam, simultaneamente, à compreensão de diversos proble mas (anemia, deficiência de vitamina A edeiodo, desnutrição, sobrepeso e obesidade), os quais implicariam em modelos explicativos diferentes, não podendo, portanto, responder com a mesma propriedade aos variados fins da pesquisa.

Por outro lado, embora a explicação de cada problema nutricional reclame, conceitualmente, a compreensão da situação alimentar que, por sua vez, implica também num modelo complexo de determinação, são raros os estudos, no Brasil eno mundo, que incluem o consumo dealimentos com a desejada pertinência e acurácia. No banco de dados da II PESN, o consumo alimentar foi levantado individualmente para crianças menores de dois anos, enquanto as idades maiores faziam parte do consumo agregado de suas famílias. Desta forma, fica impossibilitado o resgate do consumo alimentar e seu valor nutricional para o conjunto amostral de menores de cinco anos. Esta, aliás, é uma limitação queseaplica a outras análises ( na realidade, à sua quase totalidade) dos estudos analíticos sobre o estado nutricional de crianças brasileiras ${ }^{14-16}$.

Em razão de sua natureza multicausal, os déficits nutricionais em crianças, a exemplo da DEP, devem ser considerados em função de diversos fatores, como a renda familiar, ocupação, escolaridade, fatores ambientais (tipo de moradia, condições de saneamento, etc.), aspectos reprodutivos (idade da mãe, número e ordem de nascimento, intervalo interpartal) e de situações mais imediatas como o consumo de alimentos, valor da dieta, doenças concorrentes, acesso às ações de saúde e outros fatores. Esta abordagem tem sido seguidamente influenciada, ainda que escassamente aplicada, na interpretação do estado nutricional de crianças no Brasil14-16.

Ainda a respeito de aspectos metodológicos, foi utilizado o padrão de referência do $\mathrm{NCHS}^{12}$, considerando-se a necessidade de comparações com estudos publicados em meados da década de 90 no Estado de Pernambuco ${ }^{7}$, e em outros estados do Nordeste (Sergipe e Ceará) e da própria Região ${ }^{17}$. Em termos de análise "baseline", 0 presente estudo estabelece um plano de referências temporais que será desenvolvido com os dados de um novo inquérito já realizado (2006), mas só preliminarmente relatado como informe descritivo ${ }^{6}$, ao CNPq e ao M inistério da Saúde, quando também deverá ser usado o novo padrão antropométrico da OMS (2006) ${ }^{18}$.

A modelagem hierarquizada dos fatores que intervêm, de forma direta ou indireta, na deter- minação do estado nutricional, sobretudo em crianças, representa um avanço considerável no entendimento das situações analisadas. Possibilita compreender o elevado grau de colinearidade entreas diversas variáveisque agem nosecossistemas da nutrição, eo caráter circunstancial erelativo dos diversos fatores que atuam deforma autônoma ou combinada na sua determinação ${ }^{14-16}$.

O utros aspectos importantes a serem considerados são as limitações da medição de alguns fatores avaliados, tendo em vista a não existência deindicadores de referência, deinstrumentostestados em estudos epidemiológicos e ausência de pontos de corte com sensibilidade e especificidade conhecidas ${ }^{14}$. 0 momento de instalação do problema pode refletir situações distintas quanto à ocorrência do déficit estatural. Em crianças menores de dois anos, este déficit pode refletir, com razoável aproximação, o estado nutricional atual, isto é, a criança pode estar enfrentando um atraso no crescimento potencialmentereversível. Já em crianças maiores, a baixa estatura passa a ser um reflexo de déficit de crescimento no passado, de difícil reversão ${ }^{19}$. O sexo também poderá influenciar no resultado da avaliação, verificando-se, em nosso meio, uma tendência de maior ocorrência de desnutrição em crianças do sexo masculino ${ }^{20}$.

A renda familiar per capita desempenha um papel de granderelevância em relação ao risco de desnutrição, representando, portanto, uma questão primordial a ser considerada. Por motivos bem evidentes, a influência que a renda familiar exerce sobre o crescimento infantil também tem sido sistematicamente considerada na literatura internacional ${ }^{13,21}$. Sabe-se que a sua influência sobre o estado de saúde e nutrição da criança pode ser mediada e, portanto, relativizada por outros determinantes, como o acesso a serviços de saúde, educação e saneamento. Assim, seu poder de explicação tendea ser maior nas regiões menos desenvolvidas, onde a oferta de bens e serviços é restrita. Do poder aquisitivo das famílias dependem, por exemplo, a disponibilidade de alimentos, a qualidade da moradia e o acesso a serviços essenciais como os de saneamento e os de assistência à saúde, fatores de crucial importância na determinação do estado nutricional ${ }^{22,16}$.

A escolaridade materna, analisada neste estudo como um dos determinantes mais distais do estado nutricional, se mostrou fortemente associada com o retardo de crescimento das crianças. 0 grau de escolaridade pode atuar na determinação do problema, desde que esteja diretamente relacionado a uma melhor compre- 
ensão da prática de cuidados infantis ${ }^{14}$, além de que um maior nível de instrução estaria contribuindo para melhor oportunidade de emprego e melhor nível salarial. A presentando-secomo um fator em grande parte independente da renda familiar per capita, a condição de filhos de mães com menos de quatro anos de escolaridade e/ou sem instrução, aumenta significativamente as chances de retardo de crescimento nas crianças. Este papel decorre dos cuidados preventivos e curativos que a mãe realiza com mais propriedade, por dispor demais conhecimento e/ou acesso a serviçosincluindo o planejamento familiar (número de filhos e intervalo interpartal) que exercem um impacto notável sobre osindicadores de saúde e de nutrição das crianças ${ }^{10,14-16}$. 0 papel da educação feminina e os padrões reprodutivos também se mostraram fortemente associados com o crescimento das crianças, com aumentos de 1,4, 1,7 e 4,3 vezes do risco de retardo estatural quando a escolaridade da mãe era relativa à não conclusão do primeiro grau, segundo grau e inferior à escolaridadeelementar, respectivamente.

Os resultados do presente estudo demonstraram a forte associação entre o peso ao nascer e a desnutrição, constituindo um expressivo fator de risco para um posterior retardo no crescimento das crianças, na medida em que funciona como um preditor deconsiderável acuidade para o prognóstico do estado de nutrição do recémnascido ${ }^{15,16}$. Em termos estatísticos, sua importância seria ainda maior se os estudos de prevalência da desnutrição não fossem expressivamente afetados pelo viés de sobrevivência, derivado do fato de que deixam de ser contabilizadas, evidentemente, as mortes associadas ao baixo peso ao nascer ${ }^{23}$. Este, em nosso estudo, se apresentou fortemente associado com o retardo estatural das crianças, chamando a atenção às altas prevalências de déficit nutricional naquelas com peso insuficiente $(29,3 \%)$ e/ou baixo $(34,6 \%)$; chamando a atenção que, no modelo ajustado, 0 baixo peso e o peso insuficiente chegaram a aumentar seus riscos relativos em relação aos resultados das análises univariadas.

A caracterização das condições de habitação representa um bom preditor do estado nutricional. Parte-se da pressuposição bem fundamentada de que famílias pobres geralmente são mais numerosas, implicando que o número de pessoas que coabitam num mesmo espaço pode se constituir num sinalizador de risco para a desnutrição. As variáveis relativas ao domicílio podem ser conceituadas como um conjunto decausas intermediárias em relação à determinação do estado nutricional do indivíduo, sendo influenciadas pelas condições socioeconômicas. A importância do ambientedomiciliar na determinação do estado nutricional infantil foi ressaltada em alguns estudos ${ }^{14-16}$ que evidenciaram a relação entre o déficit de altura/idade com o tipo de residência da criança e o número de pessoas por dormitório. N este sentido, destaca-se o estudo de Guimarães ${ }^{20}$, queanalisando o efeito dos principais fatores associados à estatura baixa de pré escolares, em uma cidade do interior paulista, identificou que o tipo de acabamento da casa, 0 número decômodos no domicílio e o número de quartos, dentre outros, mostraram-se associados estatisticamente ao problema. Em nosso estudo, se convencionou que a condição de menos de um morador/cômodo seria a situação de re ferência, verificando-se queas demais condições (um ou mais moradores) apresentaram maiores chances de indicar crianças com déficit de estatura, com um comportamento de tendêncialinear bem evidente. Esta variável também se apre sentou como um fator de risco independente para a desnutrição, após o ajuste dos fatores econômicos, possivelmente por influenciar os padrões de morbidade, principalmente de infecções ${ }^{10}$. A condição "maiores famílias, menores habitações", muito comum nos países pobres, pode ser a imagem representativa desta situação e dos riscos nutricionais decorrentes ${ }^{24}$.

O saneamento básico exerce uma influência significativa na determinação deindicadores nutricionais, contribuindo para uma melhor ou pior situação de saúde da população ${ }^{13,25}$. A precariedade dessas condições potencializa os riscos de doenças infecto-parasitárias, as quais, por sua vez, expõem as crianças desnutridas a um processo de agravamento do estado nutricional de forma rápida e marcante ${ }^{26}$. A interferência do abastecimento de água pode se relacionar com os riscos à saúde, a partir de três efeitos principais: por levar a comunidade a consumir água de fontes alternativas de abastecimento, por induzir práticas precárias de higiene das criançase por eventual participação na contaminação da água de abastecimento na rede de distribuição ${ }^{25}$. No Brasil, mais da metade da população não conta com redes para coleta de esgotos e $80 \%$ dos resíduos gerados são lançados diretamente nos rios, sem nenhum tipo de tratamento ${ }^{27}$. Em Pernambuco, existe ainda um déficit na rede de abastecimento de água da ordem de $20 \%$, retratando, sobretudo, a situação da zona rural ${ }^{5}$.

No período de 1995 a 2000, verificou-se no Brasil uma redução de $23 \%$ da mortalidade in- 
fantil, bem como das taxas de desnutrição e de internações por diarreia, que apresentou uma queda de $37 \%{ }^{28}$. De acordo com a Pesquisa N acional de Demografia e Saúde ${ }^{9}$, observou-se um declínio na prevalência de diarreia (de 14\% em 1996 e de 9\% em 2006) e de tosse e respiração rápida (47\% em 1996 e 35\% das crianças em 2006). Em Pernambuco (2006), em relação às últimas duas semanas, a frequência referida do problema foi de $17 \%$ para o total da amostra, com os valores extremos de prevalência de $20 \%$ nas crianças do meio rural e do interior urbano, ede $10 \%$ na Região M etropolitana de Recife. N os sete dias anteriores à entrevista, 52\% das crianças apresentaram queixas de tosse, com maior incidência nas zonas urbanas ${ }^{5}$.

A visita do agente comunitário de saúde constitui um fator associado estatisticamente com 0 retardo estatural, levando à conclusão de que crianças que não recebiam sua visita estavam "protegidas" do problema. Para entender este aparente paradoxo, vale a pena considerar alguns aspectos. $\mathrm{O}$ agente comunitário de saúde tem entre suas atribuições específicas a identificação de indivíduos e de famílias expostas a situações derisco e a orientação destas para a utilização adequada dos serviços de saúde, encami- nhando-as e até agendando consultas e exames, quando necessário. Em função desse papel, é possível que sejam justificadas pelo fato das crianças de maior risco serem aquelas prioritariamente visitadas, de forma que a própria cobertura dos agentes corresponderia a um mapeamento das áreas mais pobres.

A importância singular ou autônoma de cada condicionante e, sobretudo, os efeitos de interação sinérgica ou antagônica, resultam em situações próprias para cada tempo e espaço geográfico. Assim, não existe um modelo causal único que possa ser aplicado, de forma pertinente, à explicação dos problemas nutricionais ${ }^{2}$. Na mesma população, mas em tempos diferentes, a natureza, a ordem de importância e a forma de articulação dos fatores que integram a rede causal dos problemas nutricionais podem variar de forma significativa. É possível, face ao rápido processo detransição nutricional que ocorre no Brasil, no Nordeste e, por extensão, no Estado de Pernambuco ${ }^{10,11}$ que entre as décadas de 90 e de 2000 (com projeção para 2010) tanto a prevalência do retardo estatural como seus fatores de risco tenham mudado consideravelmente, justificando, portanto, o papel atribuído a este estudo "baseline", sobretudo em seus aspectos analíticos.

\section{Colaboradores}

A Rissin, JN Figueiroa, M HA Benício eM Batista Filho participaram igualmente de todas as etapas da elaboração do artigo.

\section{Agradecimentos}

Ao M inistério da Saúde (Coordenação Geral da Política deAlimentação eN utrição), Universidade Federal de Pernambuco (Departamento de Nutrição), Secretaria de Saúde do Estado de Pernambuco e Instituto de M edicina Integral Professor Fernando Figueira, responsáveis pelo planejamento e operacionalização da II PESN, por possibilitar acesso aos dados. 
1. Bryce J, El Arifeen S, Parkyo G, Lanata C, Gwatikin D, Habicht JP. Reducing child mortality: can public health deliver? Lancet 2003; 362(9378):159-164.

2. Beghin I, Cap M, Dujardin B. Guia para evaluar el estado de nutrición. Washington, DC.: OPS (Publicación Científica, 515); 1989.

3. Instituto Brasileiro de Geografia e Estatística (IBGE). $M$ etodologia do estudo nacional de despesa familiar. Endef. Núcleo de banco de dados de informações Endef. [mimeo] Rio de Janeiro: IBGE; 1977.

4. Instituto Nacional de Alimentação e Nutrição (INAN). Pesquisa nacional de saúde e nutrição (PN SN, 1989) : resultados preliminares. Brasília: INAN; 1990.

5. Batista Filho M, Romani SAM, organizadores. Alimentação, nutrição e saúde no Estado de Pernambuco. Recife: Instituto Materno Infantil de Pernambuco (IMIP). Serie Publicações Científicas do Instituto $M$ aterno Infantil de Pernambuco (IMIP). n.7.2002; $153 \mathrm{p}$.

6. Departamento de Nutrição/U niversidade Federal de Pernambuco/Instituto $M$ aterno Infantil Prof. Fernando Figueira/ Secretaria Estadual de Saúde de Pernambuco. III Pesquisa Estadual de Saúde e Nutrição (III PESN-2006). Síntese dos resultados. Recife, 2008. [acessado 2008 out 03]. Disponível em: <http:/ /pesnpe2006. blogspot.com/2008/09/apresentao-dapesquisa. html>. Acesso em: 3 out. 2008.

7. Fundo das Nações Unidas para a Infância (UNICEF) Pernambuco. Secretaria de Saúde. Crianças e adolescentes em Pernambuco: saúde, educação e trabalho. Recife: UNICEF; 1992. 154 p.

8. Sociedade Civil Bem Estar Familiar no Brasil (Benfam). Pesquisa Nacional sobre Demografia e Saúde. Programa de pesquisas de demografia e saúde. Rio de Janeiro; 1996.

9. Brasil. M inistério da Saúde. Secretaria de Ciência, Tecnologia e Insumos Estratégicos. Departamento de Ciência e Tecnologia. Pesquisa Nacional de De mografia e Saúde da Criança e da Mulher. Brasília: M inistério da Saúde; 2008. $12 \mathrm{p}$.

10. Batista Filho M, Rissin A. A transição nutricional no Brasil: tendências regionais e temporais. $C$ ad Saude Publica. 2003; 19 (Supl.1); 181-191.

11. M onteiro CA, M ondini L, Souza ALM, Popkin BM . Da desnutrição para a obesidade: a transição nutricional no Brasil. In: Monteiro, C.A, organizador. Velhos e novos males da saúde no Brasil: a evolução do país e de suas doenças. São Paulo: Hucitec; 2000. p. 247-255.

12. National Center for H ealth Statistics (NCHS). Growth curves for children birth-18 years. Washington, D.C.: Governamment Print Office. Vital Health Statistics Series 1977; 11(165):1-74.

13. Victora CG, Fuchs SC, Flores JAC, Fonseca W, Kirkwood B. Risk factors for malnutrition in Brazilian children. The role of social and environmental variables. Bull World Health Organization 1986; (64):299309.

14. Carvalhaes MABL, Benício MHD'A. Capacidade materna de cuidar a desnutrição infantil. Rev Saude Publica 2002; 36(2):188-197.
15. Olinto MTA, Victora CG Barros, FC Tomasi E. De terminantes da desnutrição infantil em uma população de baixa renda: um modelo de análise hierarquizado. Cad Saude Publica 1993; 9(Supl. 1):14-27.

16. Rissin A, Batista Filho M, Benício, MHD'A. Condições de moradia como preditores de riscos nutricionais em crianças de Pernambuco. Rev. Bras. Saúde M atern. Infant. 2006; 6(1):59-67.

17. Fundo das Nações Unidas para a Infância (UNICEF). Saúde e nutrição das crianças nordestinas. Pesquisas estaduais 1987-1992. Brasília: UNICEF; 1995. $78 \mathrm{p}$.

18. World Health Organization (WHO). WHO Child Growth Standards: lenght/height-for-age, weightfor-age, weight-for-lenght, weight-for-height and body mass índex-for-age. M ethods and development. WHO [nonserial publication]. Geneva, Switzerland: WH O; 2006.

19. Pelletier DL. Potentiating effects of malnutrition on child mortality: epidemiologic evidence and policy implications. Food Nut Bull. 1995; 16:206-213.

20. Guimarães LV, Latorre M RDO, Barros M BA. Fatores de risco para a ocorrência de déficit estatural em pré-escolares. Cad Saude Publica 1999; 15(3):605-615.

21. Crooks, DL. Child growth and nutritional status in a high-poverty community in eastern Kentucky. Am J Phys Anthropol 1999; 109(1):129-142.

22. Monteiro,CA, Freitas ICM . Evolução de condicionantes socioeconômicos da saúde na infância na cidade de São Paulo (1984-1996). Rev Saude Publica 2000; 34(Supl. 6):8-12.

23. Oliveira O, Taddei JAAC. Efeito dos vieses de sobrevivência nas prevalências da desnutrição em crianças no sexto ano de vida. Brasil. PNSN, 1989. Cad Saude Publica 1998; 14(3):493-499.

24. Organização das Nações Unidas para a Agricultura e a Alimentação (FAO). M anejo de projetos comunitários de alimentação e nutrição: guia didático. 2000. Recife: Ministério da Saúde; 2000.

25. Teixeira JC, Heller, L. Fatores ambientais associados à desnutrição infantil em áreas de invasão, Juiz de Fora, M G. Rev. Bras. Epidemiol 2004; 7(3):270-278.

26. Bittencourt AS, Leal MC, Gadelha AMJ, Oliveira MA. Crescimento, diarreia e aleitamento materno: o caso da Vila do João. Cad Saude Publica 2003; 9 (Supl.1):7-13.

27. Instituto Brasileiro de Geografia e Estatística (IBGE). Censo Demográfico 2000. Rio de Janeiro: IBGE; 2001.

28. Brasil. M inistério da Saúde. Programa Avança Brasil. Saúde da Criança e Aleitamento M aterno. [acessado 2009 jan 22]. Disponível em: http:// www. abrasil.gov.br

Artigo apresentado em 01/06/2009

Aprovado em 03/08/2009

Versão final apresentada em 18/08/2009 